\title{
Electroless plating as surface finishing in electronic packaging
}

\begin{abstract}
Electroless plating is one type of surface finish deposition method in electronic packaging. With advancements in electronic products, the industry is shifting toward this process based on its advantages. However, the electroless plating process itself is unstable. Its stability fully depends on the substrate material, the pretreatment process, the type of solution used, and the $\mathrm{pH}$ and temperature during plating. In this article, focus is given to the three different types of electroless plating formulations used in electronic packaging that have been studied by previous researchers: electroless nickel, electroless palladium, and electroless gold.
\end{abstract}

